\title{
Resuming endoscopy during COVID-19 pandemic: ESGE, WEO and WGO Joint Cascade Guideline for Resource Limited Settings
}

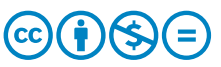

\begin{abstract}
Authors
Giulio Antonelli¹,2,3, John Gásdal Karstensen ${ }^{4,5}$, Purnima Bhat ${ }^{6}$, Uchenna ljoma7, Chukwuemeka Osuagwu ${ }^{8}$, Hailemichael Desalegn ${ }^{9}$, Hanna Abera ${ }^{7}$, Claire Guy ${ }^{10}$, Peter Vilmann ${ }^{11}$, Mario Dinis-Ribeiro ${ }^{12}$, Thierry Ponchon ${ }^{13}$, Luis Carlos Sabbagh ${ }^{14}$, Nonthalee Pausawasdi ${ }^{15}$, Govind Makharia ${ }^{16}$, Cesare Hassan ${ }^{1}$, Andrew Veitch ${ }^{17}$, Lars Aabakken*, 18 , Alanna Ebigbo*,7, for the Endoscopy and COVID-19 Cascade Working Group, European Society of Gastrointestinal Endoscopy, World Endoscopy Organization and World Gastroenterology Organization
\end{abstract}

Institutions

1 Gastroenterology Unit, Nuovo Regina Margherita Hospital, Rome, Italy

2 Department of Translational and Precision Medicine, "Sapienza" University of Rome, Italy

3 Gastroenterology and Digestive Endoscopy Unit, Ospedale dei Castelli (N.O.C.), Rome, Italy

4 Gastro Unit, Hvidovre Hospital, Hvidovre, Denmark

5 Department of Clinical Medicine, University of Copenhagen, Copenhagen, Denmark

6 ANU Medical School/Canberra Hospital -Gastroenterology, Garran, Australia

7 III Medizinische Klinik, Universitatsklinikum Augsburg, Augsburg, Germany

8 Gastroenterology Unit, Department of Medicine, University of Nigeria Teaching Hospital, Ituku-Ozalla, Enugu, Nigeria

9 Department of Internal Medicine, St. Paul's Hospital Millennium Medical College, Addis Ababa, Ethiopia

10 European Society of Gastrointestinal Endoscopy Munich, Germany

11 Gastro Unit, Copenhagen University Hospital Herlev Herlev, Denmark

12 CIDES/CINTESIS, Faculty of Medicine, University of Porto, Porto, Portugal

13 Hepatogastroenterology, Edouard Herriot Hospital, Lyon, France

14 Department of Gastroenterology, Reina Sofia and Colombia University Clinics, Bogota, Colombia

15 Faculty of Medicine Siriraj Hospital, Mahidol University, Bangkok, Thailand
16 All India Institute of Medical Sciences, New Delhi, India

17 Department of Gastroenterology, Royal Wolverhampton NHS Trust, UK

18 Department of Transplantation Medicine, Faculty of Medicine, Oslo University Hospital - Rikshospitalet, Oslo, Norway

submitted 28.1.2021

accepted after revision 23.2.2021

\section{Bibliography}

Endosc Int Open 2021; 09: E543-E551

DOI 10.1055/a-1400-9135

ISSN 2364-3722

(C) 2021. The Author(s).

This is an open access article published by Thieme under the terms of the Creative Commons Attribution-NonDerivative-NonCommercial License, permitting copying and reproduction so long as the original work is given appropriate credit. Contents may not be used for commercial purposes, or adapted, remixed, transformed or built upon. (https://creativecommons.org/licenses/by-nc-nd/4.0/)

Georg Thieme Verlag KG, Rüdigerstraße 14,

70469 Stuttgart, Germany

Corresponding author

Giulio Antonelli, Digestive Endoscopy Unit, Nuovo Regina

Margherita Hospital, Department of Translational and

Precision Medicine, Sapienza University of Rome,

Gastroenterology and Digesive Endoscopy Unit, Ospedale

dei Castelli Hospital, Ariccia, Rome, Italy

Fax: +390693278794

giulio.antonelli@gmail.com

\section{Introduction}

The ongoing COVID-19 pandemic has forced endoscopy units to stop or markedly reduce all elective endoscopic procedures and has consequently contracted endoscopic capacity throughout the world, with growing concern for a mid- and long-term 
increase in the burden of gastrointestinal diseases [1-4]. The reopening of endoscopic services is crucial to resume elective procedures but must be balanced with the need to protect healthcare personnel who are already over-represented in terms of COVID 19 morbidity and mortality [5-7].

Resumption of partial or full endoscopy capacity depends on implementation of several interventions, such as availability of Personal Protective Equipment (PPE), COVID-19 testing, distancing and separation according to the level of infection, use of telemedicine, availability of vaccines and others. At least some of these interventions are resource-consuming, representing a limitation in developing countries [8].

The European Society of Gastrointestinal Endoscopy (ESGE), the American Society of Gastrointestinal Endoscopy (ASGE) and the British Society of Gastroenterology (BSG) have all issued position statements providing guidance and recommendations for the resumption of endoscopic activity following peaks/ waves of COVID-19 [5, 6, 9, 10]. The majority of recommendations in the position papers are based on expert opinions and early survey-based or observational evidence. Many recommendations are resource-sensitive and may be unavailable in low-resource settings due to issues such as extensive costs, personnel unavailability, lack of sufficient healthcare professional training and logistical limitations $[8,11]$.

At the time this paper was drafted, nearly 3 million cases and 70000 coronavirus-related deaths had been reported in the African Continent, with the majority of states still reporting a high rate of community transmission [12]. Furthermore, the availability and the access to COVID-19 vaccination in African countries may be limited [13].

The European Society of Gastrointestinal Endoscopy (ESGE) and the World Gastroenterology Organization (WGO) have been publishing Cascade guidelines aiming to apply existing data and adapt existing guidelines for use in resource-limited settings [14-19]. This Cascade guideline is the result of a joint effort of ESGE, WEO and WGO, aiming to standardize guidance for resumption of gastrointestinal endoscopy in the different phases of the COVID-19 pandemic also in resource limited settings.

\section{Methods}

The methodology of the cascade guidelines has previously been described in an ESGE position paper [17]. Briefly, statements of the ESGE, ASGE and BSG guidelines were extracted in a dedicated sheet. Partially or totally overlapping recommendations were merged to create a single body of statements.

Following this step, members of the ESGE International Affairs Working Group (IAWG), of WEO and of WGO independently categorized the statements as resource sensitive or not. Those with an agreement of $50 \%$ or more for being resource sensitive were selected for the revision process and subsequently, adaptions were suggested for the four previously defined resource levels ( $>$ Table $\mathbf{1}$ ).

The modified statements were then subject to a Delphi process with expert doctors from low- and medium-income Countries (LMIC), where a rate of agreement of $75 \%$ or higher of all adaptions for all resource levels led to acceptance of the Cascade statement [17]. Experts from LMIC were contacted based upon contact lists of all three societies (ESGE, WEO, WGO). If a $75 \%$ agreement was not reached, the statement was subject to another round of modification before a final Delphi process was carried out.

\section{Results}

\section{Cascade statements}

\section{Statement selection}

All statements of the three original position papers were extracted to a dedicated excel sheet. Similar or overlapping statements were merged, and the statements were categorized in broad subsections. Overall, 46 statements resulted from this process, of which 21 statements were selected as resource sensitive by the working group. For this analysis, resource levels III and IV were merged. Three adapted cascade statements - one for each level - were created for each of the original recommendations, making a total of 63 adapted cascade guideline statements.

\section{$>$ Table 1 Level of treatment.}

I: Basic Core resources or fundamental services absolutely necessary for an endoscopy care system to function. By definition, a health care system lacking any basic level resource would be unable to provide endoscopic service to its patient population. It includes diagnostic procedures (gastroscopy and colonoscopy) as well and fundamental monitoring abilities (blood pressure, basic blood biochemistry).

II: Limited Second-tier resources or services that produce major improvements in outcome, such as increased survival, but that are attainable with limited financial means and modest infrastructure. It includes minor endoscopic procedures to improve major clinical outcomes (i. e. sclerotherapy/adrenaline injection, band ligation, plasma expanders, basic surgical interventions).

III: Enhanced Third-tier resources or services that are optional but important. Enhanced-level resources may produce minor improvements in outcome but increase the number and quality of therapeutic options. Most procedures that improves clinical outcome are available (i. e. biliopancreatic endoscopy, electrosurgical unit, polypectomy/mucosectomy, anaesthesia back-up).

IV: Maximal High-level resources or services that may be used in some high-resource countries or be recommended in guidelines that assume unlimited resources. To be useful, maximal-level resources typically depend on the existence and functionality of all lower-level resources. 
Delphi process

Overall, 17 experts from 9 countries participated in the Delphi process expressed their degrees of agreement for each of the recommendations. Details of the participants are provided in - Table 2. A $\geq 75 \%$ agreement was achieved for 16 of 21 proposed adaptations.

Five cascade recommendations failed to achieve the $\geq 75 \%$ agreement level. The main points of disagreement among the participants regarded the availability and use of PPE and of COVID screening and testing. In detail, the use of pre-procedural testing was said to be often unavailable even for tertiary centers, except when a high risk of transmission is suspected. In addition, an excessive time lag between testing and procedure was seen as falsely reassuring and discouraged. These statements were revised and adaptation was extended to Level II, but not to Level III as it was decided that whenever possible this strategy should be nonetheless recommended. Some participants pointed out the custom of re-using clean PPE or the washing and sterilizing of used PPE.

\section{Cascade adaptation}

Each original recommendation with the accepted adaptations is reported in $>$ Table 3 . Original statements were divided in the following domains:

1. General recommendations

2. Practical recommendations

a) Patient and staff protection, PPE use, infection prevention and control

b) COVID-19 screening and testing

c) Procedure scheduling

d) COVID-19 "minimized" units

For the cascading, it was assumed that basic endoscopy is available at all levels of care.

Limitations in PPE-availability, lack of testing capacity prior to endoscopy and infrastructural deficits regarding room space and contact tracing will impact on reopening of centers and resumption of endoscopy activity.

Most centers in low and middle-resource regions perform manual reprocessing of endoscopes. This practice, if done properly with all precautionary measures, will not be expected to lead to a higher risk of COVID-19 infections in staff involved in reprocessing. Since the virus is easily destroyed by soaps and alcohol, cross contamination of patients is also unlikely.

However, added to availability of endoscopy, some specific resources influenced the adaptation of the original guidelines and can be categorized as follows:

\section{1) Personal Protective Equipment}

The availability of PPE is a barrier for level I and II. Thus, singleuse PPE may be reutilized for more than one procedure. In the case of lack of availability of N95, the use of surgical mask is recommended. Alternatively, the use of cloth masks may be an option when surgical masks are unavailable. Methods to sterilize single-use PPE are in use in certain settings. WGO has produced guidance for use of PPE in low resource settings [20].
- Table 2 Characteristics of the participants in the Delphi Process.

\begin{tabular}{|l|l|}
\hline Geographical area & $\begin{array}{l}\text { Number of participants } \\
\text { N (\%) }\end{array}$ \\
\hline Algeria & $1(5.8 \%)$ \\
\hline Ethiopia & $4(23.5 \%)$ \\
\hline France & $1(5.8 \%)$ \\
\hline Kenya & $1(5.8 \%)$ \\
\hline Morocco & $2(11.7 \%)$ \\
\hline Mozambique & $1(5.8 \%)$ \\
\hline Nigeria & $3(17.6 \%)$ \\
\hline Senegal & $1(5.8 \%)$ \\
\hline Tunisia & $3(17.6 \%)$ \\
\hline
\end{tabular}

\section{2) Triage and tracing}

Due to infrastructure issues and remote location of patients from hospitals, contact between the health centre and the patients before endoscopy for triage and/or testing is often not feasible in levels I and II. In this case, it has been recommended to triage patients on the day of endoscopy at least for symptoms and signs. Similarly, a systematic policy of triage according to symptoms/signs should be recommended to healthcare professionals (HCPs). At a similar level, a policy of systematic tracing of patients after procedures is not available, and may be replaced by instructing patients to notify whenever symptoms appear in the days following the endoscopic procedure. In case patients report symptoms suggestive for COVID and no testing is available, these cases should be considered positive for COVID.

\section{3) Medical and non-medical staff}

Most services in developing countries are short-staffed, and this may be worsened by redeployment of endoscopy manpower to COVID areas. In addition, part of the staff should be redirected to tasks of pre-procedural risk assessment. To minimize infection risk, a possible stratification may be proposed with procedures at low-risk of viral transmission to be allocated in one day/session, and the others in different days/sessions ("COVID-minimized" days/units). Ideally, HCPs should rotate in a fixed way so that only those exposed should be removed in case of transmission. However, when organizing COVID "minimized" units/days, staff availability should be taken into account.

\section{4) Infrastructure}

Health facilities in levels I and II face space issues to apply social distancing. Family attendance should be avoided whenever possible. The proposal of COVID "minimized" days, where only low-risk patients and procedures are scheduled may be a more viable alternative than COVID-minimized areas in the same unit, in units where space is a limiting factor. 
- Table 3 Adapted recommendations according to level of treatment care.

\section{General Recommendations}

Resumption of endoscopy services is critically dependent on the availability of PPE

Choice of PPE level should be determined by patient risk stratification, the nature of the proposed procedure and the results of patient testing.

Infection Prevention and Control (IPC) interventions must be tailored to the local availability and affordability of resources, while keeping in consideration the local prevalence of COVID-19 and community viral transmission rates.

Given the lack of high-level evidence, the exclusive use of serology or rapid antigen-testing for pre-endoscopy patient triage cannot be recommended at this time.

A return to full gastrointestinal endoscopy procedure capacity should be pursued in those areas without evidence of community transmission of COVID-19, while continuing to adhere to IPC measures.

Gastrointestinal endoscopy units involved in endoscopy training and research activities may gradually restart their endoscopy training programs and research activities, provided this will not further delay needed gastrointestinal endoscopic procedures.

No changes are recommended to established reprocessing procedures for endoscopes and accessories. Standard bedside pre-cleaning, followed by manual cleaning and high-level disinfection in the reprocessing facility should continue.

No changes are recommended to 'terminal cleaning' procedures for cleaning and disinfecting the endoscopy unit at the end of the day.

In areas with limited endoscopic capacity, scheduling of patients should be prioritized reflecting the potential of serious outcomes with delay of procedures. Providers should indicate the patient's procedural tier in their telehealth visit or telephone encounter note.

Patients' fears of contracting COVID-19 infection while visiting an endoscopy unit should be properly addressed.

In "COVID Minimized" Units: Prioritizing procedures which may be less aerosol generating- flexible sigmoidoscopy and colonoscopy - as the risk of viable, transmissible virus in stool appears to be much lower

In "COVID Minimized" Units: A slower throughput of patients to reduce the risk of positive and negative patients meeting

\section{Adaptation}

- Level I: No adjustment

- Level II: No adjustment

- Level III: No adjustment

- Level I: No adjustment

- Level II: No adjustment

- Level III: No adjustment

- Level I: In case of unavailability of single-use PPE for every procedure, re-use of PPE under certain conditions may be considered

- Level II: No adjustment

- Level III: No adjustment

- Level I: No adjustment

- Level II: No adjustment

- Level III: No adjustment

- Level I: No adjustment

- Level II: No adjustment

- Level III: No adjustment

- Level I: No adjustment

- Level II: No adjustment

- Level III: No adjustment

- Level I: No adjustment

- Level II: No adjustment

- Level III: No adjustment

- Level I: No adjustment

- Level II: No adjustment

- Level III: No adjustment

- Level I: In case of telephone unavailability, prioritization can be done in the scheduling phase by the provider.

- Level II: No adjustment

- Level III: No adjustment

- Level I: No adjustment

- Level II: No adjustment

- Level III: No adjustment

- Level I: No adjustment

- Level II: No adjustment

- Level III: No adjustment

- Level I: In case of unavailability of multiple rooms, lower risk procedures may be performed in separate days ("COVID minimized" days) than high-risk procedures.

- Level II: When two endoscopy suites are available, we suggest to create a "COVID-minimized" area, combined with separated pre- and post-endoscopy waiting areas if available.

- Level III: No adjustment

\section{Practical Recommendations}

Patient and Staff Protection, PPE use, Infection Prevention and Control

Pre procedure COVID-19 questionnaire within 72 hours of visit on the telephone. Consider using risk stratification questionnaires including questions regarding fever, travel history, occupational exposure, contact history and clustering type).
- Level I: Telephone contact may be unavailable, so we suggest a risk stratification questionnaire physically on the endoscopy day. However, HCP administering the questionnaire should use highest available PPE.

- Level II: No adjustment, however, in the case of telephone unavailability for patients in rural areas, we suggest to refer to level I suggestion.

- Level III: No adjustment; however, in the case of telephone unavailability for patients in rural areas, we suggest referring to level I suggestion. 
- Table 3 (Continuation)

\section{General Recommendations}

Update of questionnaire upon arrival at facility

Patients should notify presence of any change in symptoms or condition that may occur between scheduling and procedure date.

Daily questionnaire to healthcare personnel (HCP)

Supplemental use of telehealth services can be considered

Onsite forehead temperature measurement (patients and HCP)

Appropriate social distancing of patients (and staff) needs to be addressed preand post-procedure. Possible interventions: markings at $1.5 \mathrm{~m}$ for distancing, waiting benches modifications for distancing, staff policing the waiting room and assuring distancing and PPE are implemented, etc.

\section{Adaptation}

- Level I: No adjustment

- Level II: No adjustment

- Level III: No adjustment

- Level I: No adjustment

- Level II: No adjustment

- Level III: No adjustment

- Level I: HCP should be aware of any COVID-like symptoms arising

- Level II: HCP should be aware of any COVID-like symptoms arising

- Level III: HCP should be aware of any COVID-like symptoms arising

- Level I: Telehealth services may not be available at all centers

- Level II: Telehealth services may not be available at all centers

- Level III: No adjustment

- Level I: No adjustment

- Level II: No adjustment

- Level III: No adjustment

- Level I: In case of unavailability of enough waiting room space, patients should be asked to wait outside the endoscopy room in designated areas or scheduling should be adapted to accommodate for space needs

- Level II: Appropriate social distancing of patients (and staff) needs to be addressed pre- and post-procedure. Possible interventions: markings at $1.5 \mathrm{~m}$ for distancing, waiting benches modifications for distancing, staff policing the waiting room and assuring distancing and PPE are implemented, etc.

- Level III: No adjustment

- Level I: Due to potential lack of phone and/or testing availability, patients should be educated to report to the center in case of "COVD-like" symptoms development

- Level II: Due to potential lack of phone and/or testing availability, patients should be educated to report to the center in case of "COVID-like" symptoms development

- Level III: No adjustment

If positive test of staff or patient, contact tracing should be initiated

All patients and staff should wear ear-loop surgical masks at all times when in the facility.

- Level I: In case of staff or patient positivity, local healthcare authorities should be informed and "intra-unit" contact tracing should be performed

- Level II: In case of staff or patient positivity, local healthcare authorities should be informed and "intra-unit" contact tracing should be performed

- Level III: No adjustment

- Level I: In case of unavailability of surgical masks, the use of cloth-masks can be considered

- Level II: No adjustment

- Level III: No adjustment

- Level I: No adjustment

- Level II: No adjustment

- Level III: No adjustment

- Level I: No adjustment

- Level II: No adjustment

- Level III: No adjustment

- Level I: In case of unavailability of surgical masks, the use of cloth-masks can be considered.

- Level II: No adjustment

- Level III: No adjustment 
Table 3 (Continuation)

\section{General Recommendations}

Staff PPE in pre- and post-operative area:

- Surgical/ear loop masks

- Nitrile gloves

- N95 respirator or equivalent can be considered, depending on availability, if direct patient contact, e.g. helping patients gown or dress, conducting patients out of center for discharge

PPE in operative/procedure room:

- N95 respirator or equivalent

- Nitrile gloves

- Impervious gowns, if available. Laundered gowns have replaced lightweight disposable gowns in some centers

- Face shields/eye protection

- Head covering (hair net, bouffant type or surgical cap)

\section{Adaptation}

- Level I: No adjustment

- Level II: No adjustment

- Level III: No adjustment

\section{COVID Screening and Testing}

Where possible, all outpatients being considered for endoscopy should undergo antigen testing based on molecular diagnosis (PCR or iNAAT) 1-3 days prior to their procedure
- Level I: In case of unavailability of recommended PPE, the highest level of available PPE should be employed, based on local pandemic phase, patient risk status and procedure priority

- Level II: In case of unavailability of recommended PPE, the highest level of available PPE should be employed, based on local pandemic phase, patient risk status and procedure priority

- Level III: No adjustment
A test-and-scope strategy in asymptomatic patients, where testing is negative, might be considered to save PPE.
- Level I: Pre-endoscopy testing may not be readily available or have a long turnaround time and, as such, may not be part of routine pre-endoscopy screening

- Level II: Pre-endoscopy testing may not be readily available or have a long turnaround time and, as such, may not be part of routine pre-endoscopy screening

- Level III: No adjustment

- Level I: Pre-endoscopy testing may not be readily available or have a long turnaround time and, as such, may not be part of routine pre-endoscopy screening

- Level II: Pre-endoscopy testing may not be readily available or have a long turnaround time and, as such, may not be part of routine pre-endoscopy screening

- Level III: No adjustment

- Level I: Pre-endoscopy testing may not be readily available or have a long turnaround time and, as such, may not be part of routine pre-endoscopy screening

- Level II: Pre-endoscopy testing may not be readily available or have a long turnaround time and, as such, may not be part of routine pre-endoscopy screening

- Level III: No adjustment

- Level I: No adjustment

- Level II: No adjustment

- Level III: No adjustment

In the case of limited molecular testing availability, testing should be reserved for those patients considered to be at high-risk for having COVID-19 infection.

\section{Procedure Scheduling}

The high administrative burden of telephone screening \pm antigen testing and telephone follow-up is likely to require endoscopy units to have additional administrative and clerical staff to deliver this.
- Level I: In case of unavailability of additional staff, existing staff may be reorganised to undertake pre- and post- endoscopic screening

- Level II: In case of unavailability of additional staff, existing staff may be reorganised to undertake pre- and post-endoscopic screening

- Level III: No adjustment

\section{Room Requirements and Cleaning Measures}

Reprocessing staff should be donning personal protective equipment (PPE) that includes gloves, gown, face shield, bonnet and mask (N95 if available).
- Level I: If not all recommended PPE are available, reprocessing staff should use the highest grade of PPE available in the center

- Level II: If not all recommended PPE are available, reprocessing staff should use the highest grade of PPE available in the center

- Level III: No adjustment 
- Table 3 (Continuation)

\section{General Recommendations}

EPA-registered hospital-grade disinfectant solutions and wipes should be used in procedure rooms to clean all high-touch and horizontal surfaces

\section{Adaptation}

- Level I: No adjustment

- Level II: No adjustment

- Level III: No adjustment

\section{“COVID Minimized" Units}

Linear patient flow through the unit, (no crossing of COVID positive and negative pathways, separate entrance and exit)
- Level I: Due to unavailability of multiple rooms, lower risk procedures may be performed in separate days ("COVID minimized" days) than high-risk procedures.

- Level II: When two endoscopy suites are available, we suggest to create a "COVID-minimized" area, combined with separated pre- and post-endoscopy waiting areas if available.

- Level III: No adjustment

Keeping known / suspected COVID patients out of "COVID-minimized" units (e. g. scope in theatre or at the bedside)

- Level I: Known or suspected COVID patients should be scoped separately (e. g. end of the day or "hot days").
- Level II: Known or suspected COVID patients should be scoped separately (e. g. end of the day or "hot days").

- Level III: No adjustment

- Level I: No adjustment

- Level II: No adjustment

- Level III: No adjustment COVID-positive patients in separate rooms, prioritised to the afternoon to allow deep cleaning and settling of the rooms overnight

Staff will also require enhanced viral screening to maintain "COVID-minimized" units. e. g. pre-work symptoms and fever-free confirmation; staff rotation to work between "hot" and "COVID-minimized" parts of a hospital or sites should be avoided. be possible. HCP should report any possible exposure or
- Level I: Due to shortage of trained endoscopy staff, separation between "COVID-minimized" staff and "hot" staff may not COVID-like symptoms.

- Level II: Due to shortage of trained endoscopy staff, separation between "COVID minimized" staff and "hot" staff may not be possible. HCP should report any possible exposure or COVID-like symptoms.

- Level III: No adjustment

IPC, Infection Prevention and Control; HCP, healthcare personnel; PPE, personal protective equipment

\section{5) Vaccination}

Since the societies recommendations were published, development and rollout of the first COVID-19 vaccines have commenced world-wide $[21,22]$. It is likely that more vaccine candidates will be available and the WHO has indicated a strong need to prioritise access to LMIC where populations are most vulnerable [23]. Most countries that have commenced a vaccination program have targeted vulnerable populations first, and health workers second, as initial vaccine recipients. The successful rollout of vaccines in LMIC may further enable rapid opening of endoscopy facilities, and minimise risk to staff and patients, and is strongly encouraged. However, as immunisation efficacy may be variable, and new COVID-19 strains continue to be discovered, recommendations for PPE and infection control remain unchanged, even for vaccinated staff and patients.

\section{Conclusion}

In conclusion, when summarizing international societies' recommendations regarding the resumption of endoscopy during COVID-19 pandemic, almost half of these resulted to be critically dependent on sensitive resources, primarily personal protective equipment. Using a previously validated methodology, we have adapted resource sensitive recommendations to resource limited settings, with particular regard to PPE, limited infrastructure, staff shortage and triage procedures.

The cascade adaptations presented here are in conjunction with return strategies reported previously, and which mainly included pre-screening and risk stratification based on questionnaires and temperature measurement [8]. Strategies for multiple use of PPE, especially N95 masks and water-resistant longsleeved gowns, have also been described, and may form an important part of return strategies in resource-poor regions. COVID-19 infection rates, with temporary surges in disease activity, will most likely persist; nevertheless, the risk of infection for endoscopy staff must be weighed against the benefits for patients presenting for endoscopy. Unlike in most European countries with elective endoscopic activity centered around screening programs, the indications for endoscopic procedures in resource-poor regions are usually symptom-driven, and often include alarm symptoms such as bleeding or dysphagia [17]. As such, resumption and maintenance of endoscopic activity is crucial for mortality and prognosis of gastrointestinal disorders in such settings. Reuse strategies for PPE, on-site triage of patients as well as introduction of "COVID-minimized 
days" have formed the framework of the cascade adaptations to guide HCP in resource-poor settings through the COVID-19 pandemic.

\section{Competing interests}

The authors declare that they have no conflict of interest.

References

[1] Repici A, Pace F, Gabbiadini R et al. Endoscopy units and the Coronavirus Disease 2019 outbreak: A multicenter experience from Italy. Gastroenterology 2020; 159: 363-366.e3

[2] Furnari M, Eusebi LH, Savarino E et al. Effects of SARS-CoV-2 emergency measures on high-risk lesions detection: a multicentre crosssectional study. Gut 2020: doi:10.1136/gutjnl-2020-323116

[3] Lantinga MA, Theunissen F, Ter Borg PC] et al. Impact of the COVID-19 pandemic on gastrointestinal endoscopy in the Netherlands: analysis of a prospective endoscopy database. Endoscopy 2020; 53: 166-170

[4] Zorzi M, Hassan C, Capodaglio G et al. Colonoscopy later than 270 days in a fecal immunochemical test-based population screening program is associated with higher prevalence of colorectal cancer. Endoscopy 2020; 52: 871-876

[5] Gralnek IM, Hassan C, Beilenhoff U et al. ESGE and ESGENA Position Statement on gastrointestinal endoscopy and the COVID-19 pandemic. Endoscopy 2020; 52: 483-490

[6] Gralnek IM, Hassan C, Beilenhoff $U$ et al. ESGE and ESGENA Position Statement on gastrointestinal endoscopy and COVID-19: An update on guidance during the post-lockdown phase and selected results from a membership survey. Endoscopy 2020; 52: 891-898

[7] Gralnek IM, Hassan C, Dinis-Ribeiro M. COVID-19 and endoscopy: implications for healthcare and digestive cancer screening. Nat Rev Gastroenterol Hepatol 2020; 17: 444-446

[8] Ebigbo A, Karstensen JG, Bhat $P$ et al. Impact of the COVID-19 pandemic on gastrointestinal endoscopy in Africa. Endosc Int Open 2020; 8: E1097-E1101

[9] Sawhney MS, Bilal M, Pohl H et al. Triaging advanced Gl endoscopy procedures during the COVID-19 pandemic: consensus recommendations using the Delphi method. Gastrointest Endosc 2020; 92: 535542

[10] Rees C], East JE, Oppong K et al. Restarting gastrointestinal endoscopy in the deceleration and early recovery phases of COVID-19 pandemic:
Guidance from the British Society of Gastroenterology. Clin Med Lond Engl 2020; 20: 352-358

[11] Ebigbo A, Römmele C, Bartenschlager C et al. Cost-effectiveness analysis of SARS-CoV-2 infection prevention strategies including preendoscopic virus testing and use of high risk personal protective equipment. Endoscopy 2020; 53: 156-161

[12] Outbreak Brief 51: Coronavirus Disease 2019 (COVID-19) Pandemic. Afr CDC; Im Internet (Stand 14.01.2021): https://africacdc.org/ download/outbreak-brief-51-coronavirus-disease-2019-covid-19pandemic

[13] Framework for Fair, Equitable and Timely Allocation of COVID-19 Vaccines in Africa (Highlights of Day 1). Afr CDC; https://africacdc. org/download/framework-for-fair-equitable-and-timely-allocationof-covid-19-vaccines-in-africa-highlights-of-day-1/ [14.01.2021]

[14] Ebigbo A, Karstensen JG, Aabakken L et al. Esophageal stenting for benign and malignant disease: European Society of Gastrointestinal Endoscopy (ESGE) Cascade Guideline. Endosc Int Open 2019; 7: E833-E836

[15] Karstensen JG, Ebigbo A, Bhat P et al. Endoscopic treatment of variceal upper gastrointestinal bleeding: European Society of Gastrointestinal Endoscopy (ESGE) Cascade Guideline. Endosc Int Open 2020; 8: E990-E997

[16] Karstensen JG, Ebigbo A, Aabakken L et al. Nonvariceal upper gastrointestinal hemorrhage: European Society of Gastrointestinal Endoscopy (ESGE) Cascade Guideline. Endosc Int Open 2018; 6: E1256E1263

[17] Hassan C, Aabakken L, Ebigbo A et al. Partnership with African Countries: European Society of Gastrointestinal Endoscopy (ESGE) - Position Statement. Endosc Int Open 2018; 6: E1247-E1255

[18] Fried M, Krabshuis ]. Can "Cascades" make guidelines global? J Eval Clin Pract 2008; 14: 874-879

[19] https://www.worldgastroenterology.org/guidelines/global-guidelines

[20] Leddin D, Armstrong D, Raja Ali RA et al. Personal protective equipment for endoscopy in low-resource settings during the COVID-19 pandemic: Guidance from the World Gastroenterology Organisation. J Clin Gastroenterol 2020; 54: 833-840

[21] Baden LR, El Sahly HM, Essink B et al. Efficacy and safety of the mRNA1273 SARS-CoV-2 vaccine. N Engl J Med 2021; 384: 403-416

[22] Polack FP, Thomas S], Kitchin $\mathrm{N}$ et al. Safety and efficacy of the BNT162b2 mRNA COVID-19 vaccine. N Engl J Med 2020; 383: 26032615

[23] Coronavirus disease (COVID-19): Vaccine access and allocation. https://www.who.int/news-room/q-a-detail/coronavirus-disease(covid-19)-vaccine-access-and-allocation [12.01.2021]

\section{CORRECTION}

Giulio Antonelli, John Gásdal Karstensen, Purnima Bhat et al. Resuming endoscopy during COVID-19 pandemic: ESGE, WEO and WGO Joint Cascade Guideline for Resource Limited Settings

Endoscopy International Open 2021; 09: E543-E551.

DOI: $10.1055 / a-1400-9135$

In the above mentioned article the name of the second author was spelled incorrectly. Correct is: John Gásdal Karstensen. 
- Appendix 1 Endoscopy and COVID-19 Cascade Working Group.

\begin{tabular}{|l|l|l|}
\hline Surname & Name & Country \\
\hline Titsaoui & Djamila & Algérie \\
\hline Fikadu Girma & Gudissa & Ethiopia \\
\hline Yemisrach & Chanie & Ethiopia \\
\hline Hailemichael Desalegn & Mekonnen & Ethiopia \\
\hline Paulos & Shume & Ethiopia \\
\hline thierry & ponchon & France \\
\hline Michael & Mwachiro & Kenya \\
\hline Dafr allah & Benajah & Morocco \\
\hline Mohamed & Borahma & Morocco \\
\hline Prassad & Modcoicar & Mozambique \\
\hline Chukwuemeka & Osuagwu & Nigeria \\
\hline Uchenna & ljoma & Nigeria \\
\hline Babatunde & Duduyemi & Nigeria \\
\hline Ibrahima & Diallo & Senegal \\
\hline Dorra & Trad & Tunisia \\
\hline Meriam & Sabbah & Tunisia \\
\hline Lamine & Hamzaoui & Tunisia \\
\hline
\end{tabular}

